\title{
The effect of hindbrain lesions on a variety of measures of feeding behavior in rats
}

\author{
J. M. HALPERIN, S. J. ELLMAN, Y. STERN, C. PAVLIDES, \\ R. HALPERIN, and S. S. STEINER
}

The City College of the City University of New York, New York, New York 10031

\begin{abstract}
Hyperphagia following hypothalamic (HYP) lesions appears to be due to the disruption of neural fibers ascending from more posterior structures. Direct connections exist between the locus coeruleus (LC) and subcoeruleus (SC), and several HYP nuclei. These experiments examined the effects of LC and SC lesions on food intake, taste responsivity, meal patterns, and the circadian aspects of feeding. Experiment 1 examined the effects of bilateral LC lesions on feeding in rats maintained on a sweetened milk diet. One week after the lesions, the rats became hyperphagic. Histological analyses revealed that the critical area involved in the hyperphagia was ventral to the LC. Experiment 2 examined the effects of SC lesions in rats maintained on a solid food diet. These rats became hyperphagic, but not hyperdipsic. Histological and neurochemical analyses indicate that SC, rather than LC, destruction is responsible for the hyperphagia.
\end{abstract}

Lesions placed in the area of the ventromedial hypothalamus (VMH) result in a syndrome characterized by hyperphagia, obesity, finickiness, the loss of the circadian feeding cycle, and increased meal size with little change in meal frequency (for review, see Teitelbaum, 1961). Contrary to earlier beliefs, Gold (1973) demonstrated that destruction to fibers of passage, rather than the ventromedial nucleus of the hypothalamus, was responsible for producing this syndrome. Further research indicated that the various behavioral abnormalities which make up the hypothalamic (HYP) hyperphagia syndrome may, in fact, be due to the destruction of distinct structures or pathways that converge in that area. This suggests disparate mediation of food intake, satiety, taste responsivity, and the circadian aspects of feeding. By varying the precise locus of lesions or knife cuts within, and outside of, the HYP, investigators have produced hyperphagia without finickiness or loss of circadian rhythmicity (Ahlskog, 1974; Ahlskog \& Hoebel, 1973; Graff \& Stellar, 1962), as well as increased taste responsivity with minimal hyperphagia (Graff \& Stellar, 1962; Sclafani, 1971). Moreover, the extrahypothalamic origins of the interrupted pathways appear to be from more posterior structures. While coronal knife cuts through the posterior HYP that resulted in minimal cellular damage have produced a marked hyperphagia and obesity (Grossman, 1971), more posterior knife cuts restricted to the central gray or to the ventromedial

The authors gratefully acknowledge the assistance of $R$. J. Bodnar, J. Rosen, and A. Tempel. J. M. Halperin's present address is: New York State Psychiatric Institute, 722 West 168 Street, New York, New York 10032. portions of the tegmentum have failed to alter feeding behavior (Grossman \& Grossman, 1977). Yet knife cuts placed just ventral to the central gray but dorsal to the ventro-medial tegmentum have resulted in hyperphagia and hyperdipsia. Hyperphagia has been associated with effective cuts in the dorsal lateral extent, and hyperdipsia has been associated with more ventromedial cuts (Grossman \& Grossman, 1977).

Direct connections exist between the dorsal hindbrain and several nuclei within the HYP that have been implicated in the regulation of feeding behavior (Jones \& Moore, 1977; Kobayashi, Palkovits, Jacobowitz, \& Kopin, 1975; Lindvall \& Bjorklund, 1974; for review, see Amaral \& Sinnamon, 1977). Many of these pathways, which originate in the locus coeruleus (LC) and subcoeruleus (SC), do not ascend through the dorsal noradrenergic bundle, which is the major pathway projecting from that brainstem area (Lindvall \& Bjorklund, 1974; Maeda \& Shimizu, 1972; Olson \& Fuxe, 1972).

LC lesions have been reported to result in a longterm hyperphagia in monkeys (Redmond, Huang, Snyder, Maas, \& Baulu, 1977). However, several studies that examined the effect of LC lesions on feeding in rats resulted in a temporary hypophagia followed by a rapid recovery (Amaral \& Foss, 1975; Osumi, Oishi, Fujiwara, \& Takaori, 1975; Ross, Smith, \& Reis, 1973). Unfortunately, none of these studies reported feeding behavior for more than 5 days following the lesions. Ross and Reis (1974) have reported that it takes approximately 1 week for dopamine-beta-hydroxylase (a specific marker of norepinephrine) reductions to occur in the HYP following hindbrain lesions. Research examining the 
effects of LC lesions on social behaviors in rats for 40 days following the lesions reported increased time spent feeding and increased weight gain, but actual food intake was not measured (Eison, Stark, \& Ellison, 1977).

The experiments reported here examined the effects of dorsal hindbrain lesions, in the area of the LC and SC, on a variety of measures of feeding behavior in rats. If some of the fibers whose transection were responsible for hyperphagia ascend from the dorsal hindbrain, these lesions should result in increased feeding. If the classical HYP hyperphagia syndrome was due to the disruption of several neural circuits which originate from distinct extrahypothalamic cell groups regulating various aspects of feeding behavior, then some, but not all, aspects of this syndrome should be observed with these posterior lesions.

\section{EXPERIMENT 1}

Experiment 1 examined whether bilateral lesions placed in the area of the LC altered feeding behavior of rats maintained on a sweetened milk diet. This diet was used to maximize the probability of observing lesion-induced alterations in feeding behavior based upon alterations in taste responsivity.

\section{Method}

Twelve female albino Sprague-Dawley rats, weighing approximately $300 \mathrm{~g}$, were housed individually under $12 \mathrm{~L} / 12 \mathrm{D}$ lighting conditions throughout the experiment and had ad-lib access to food and water.

The rats were anesthetized with sodium thiopental $(45 \mathrm{mg} / \mathrm{kg})$ and stereotaxically implanted with two chronically indwelling, bipolar, stainless steel electrodes which were $.5 \mathrm{~mm}$ at their widest extent and insulated except at the tips. With the incisor bar set at $-5 \mathrm{~mm}$, electrodes in eight rats were aimed bilaterally at the LC at a 16-deg angle to the midsagittal plane, $1.5-2.0 \mathrm{~mm}$ posterior to the lambda suture, $3.0 \mathrm{~mm}$ to the left and right of midline, and 7.1-7.3 mm below the surface of the skull. Two rats were implanted at the same coordinates, but at a 0 -deg angle to the midsagittal plane and $7.0 \mathrm{~mm}$ below the surface of the skull. Two additional rats received sham surgery.

After surgery, the rats were maintained individually in Plexiglas chambers. Calibrated bottles containing the sweetened milk diet ( $300 \mathrm{ml}$ Borden's Magnolia milk, $600 \mathrm{ml}$ water, $.3 \mathrm{ml}$ Poly-visol vitamins) were suspended so that the sipper tube was approximately $.5 \mathrm{~cm}$ outside a $1.4-\mathrm{cm}$-diam hole in the wall of the chamber. This method, similar to that described by Kissileff (1969), minimizes random contact with the sipper tube and prevents continuous contact with the tube during feeding. A calibrated cup was used to collect spillage. The sipper tube and floor grid of the chamber were wired so that each lick produced an individual electrical pulse. The temporal distribution of licks was recorded and scored for meal size (number of licks per meal) and meal frequency. A meal was defined as no less than 10 licks, separated by at least 10 min from any other licks. Licks that did not occur during meals were counted and recorded as nonmeal licks.

After 15 days' adaptation to the new diet, 7 days of baseline feeding behavior was recorded. The rats were then lightly anesthetized (sodium thiopental, $30 \mathrm{mg} / \mathrm{kg}$ ) and given bilateral radio frequency lesions through the chronically implanted electrodes; the shams were anesthetized. Twenty-one days of ad-lib postlesion feeding data were recorded for each rat. Subsequent to this, response to 4 days of quinine-adulterated milk (.3\% quinine sulfate) was recorded in nine of the rats. The rats were then sacrificed. Each brain was removed from the skull and placed in a $10 \%$ Formalin solution for 1 week. They were then frozen, cut in 40-micron sections, and stained with cresyl violet and luxol fast blue according to the method of Kluver and Barrera (1953). Localization and extent of lesions were determined by a judge who had no knowledge of the behavioral data. The magnitude of destruction to the LC, SC, and other adjacent nuclei was estimated by projecting an image of the lesioned tissue onto a grid. The number of boxes subsumed by lesioned tissue with respect to the area of the nucleus was determined. This was done for each rat on a minimum of three sections at different anterior-posterior levels for each structure.

The rats were grouped as bilaterally LC-lesioned or non-LClesioned. The data were broken down into baseline, Postlesion Week 1, Week 2, and Week 3, and analyzed using a profile analysis (Morrison, 1976). This analysis utilizes a HotellingLawley trace to compare, between groups, the trends of behavioral changes over time. In addition, it can be used to make several within- and between-group comparisons. These analyses were performed on changes in milk intake, total number of licks, body weight, light-dark distribution of feeding, meal patterns, and response to quinine adulteration. Pearson product correlation coefficients were determined to measure the relationship between the magnitude of change in feeding and the amount of destruction to specific hindbrain nuclei.

\section{Results}

Histology. Six rats had bilateral destruction of the LC. Of the six non-LC-lesioned rats, two had lesions lateral to the LC which destroyed parts of the medial cerebellar peduncles, the trochlear nerve, and the trigeminal spinal tract, but left the entire pontine central gray intact; two had lesions anterior and ventral to the LC, which caused destruction to the superior cerebellar peduncles and destroyed or extended ventral to the medial longitudinal fasciculus; and two were shams.

The lesions in the LC group extended to other structures beside the LC. Many of them impinged upon, or destroyed, parts of the SC, dorsal tegmental nucleus (DTN), and trigeminal motor nucleus (TMN). A few LC lesions extended laterally to the brachium conjunctivum and ventrally to the medial longitudinal fasciculus. Figure 1 shows the extent of the bilateral LC lesions in one rat. The left lesion in this figure is among the largest in the group, whereas the right is among the smallest.

Figure 2 shows the maximal extent of all the lesions for each group when overlapped with each other. The lesions of the different groups overlap anterior to the pontomesencephalic junction, but not within the pontine tegmentum.

Behavioral analyses. No significant differences in feeding behavior occurred between the three nonLC-lesioned groups, so they were combined to form the controls. Because of equipment failure in one rat's chamber, analyses using the lick data (total licks and meal pattern analyses) were based upon 11 subjects. Table 1 summarizes the within- and 


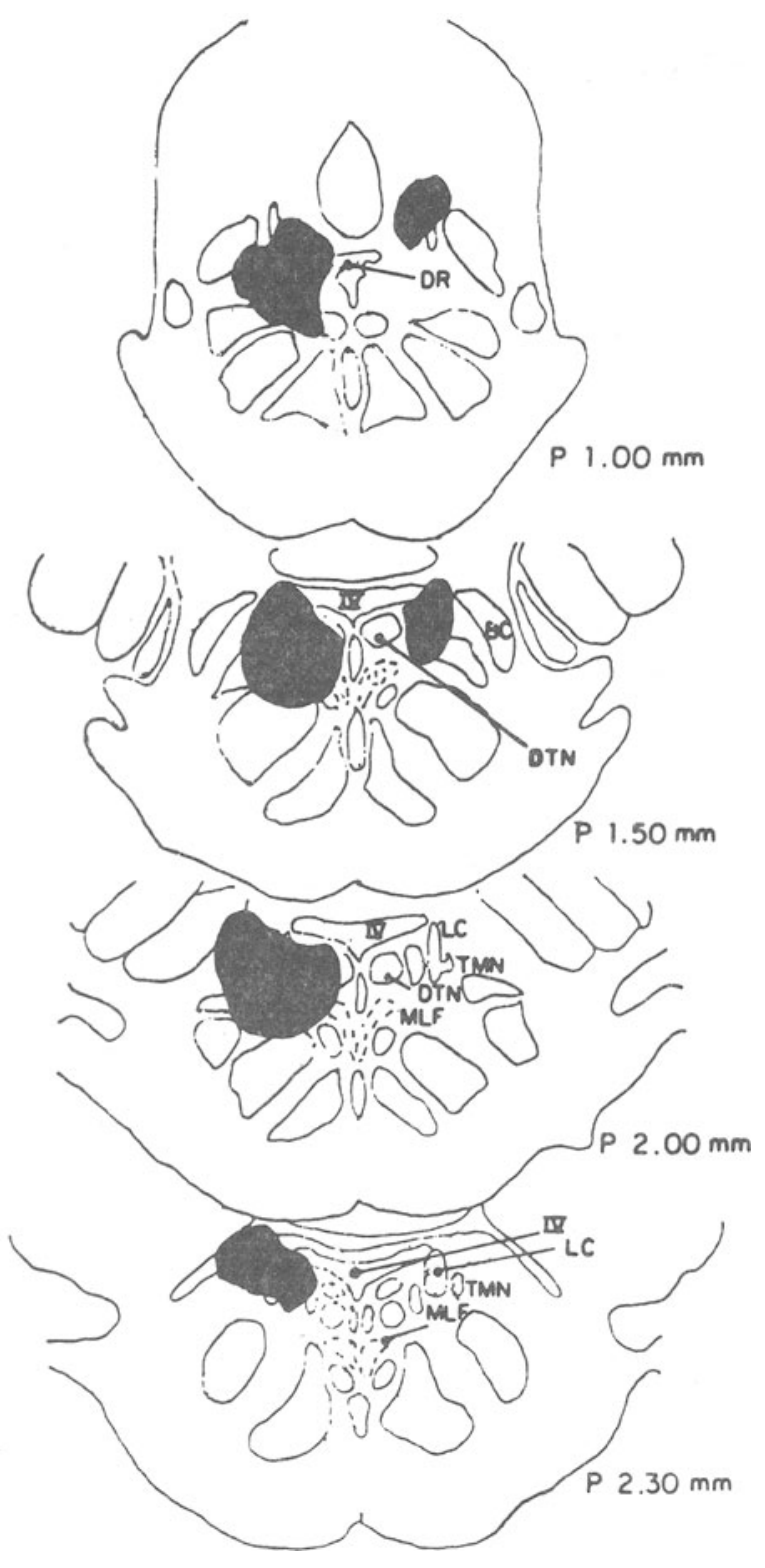

Figure 1. Schematic representation of the bilateral LC lesions in rat $84 \mathbf{J}$. LC = locus coeruleus; $T M N=$ trigeminal motor nucleus; MLF = medial longitudinal fasciculus; DTN $=$ dorsal tegmental nucleus; $\mathbf{B C}=$ brachium conjunctivum; $\mathbf{D R}=$ dorsal raphe; IV = fourth ventricle.

between-groups analyses for changes from baseline feeding during the first, second, and third weeks postlesion.

The Hotelling-Lawley trace indicated significant differences in the trends over time between the LC and control group for both milk intake $[F(3,8)=$ $11.04, \mathrm{p}<.005]$ and total number of licks $[\mathrm{F}(3,7)=$ $5.65, \mathrm{p}<.05]$. The change from baseline milk intake did not differ between groups during the 1st week postlesion, but was significantly greater in the LC group during the 2 nd and 3 rd weeks postlesion. The LC group had a significantly greater increase in number of licks during all 3 weeks postlesion. Within the LC-lesioned group, there was no significant difference in milk intake or in number of licks between baseline and the 1st week postlesion. However both of these measures increased significantly be-

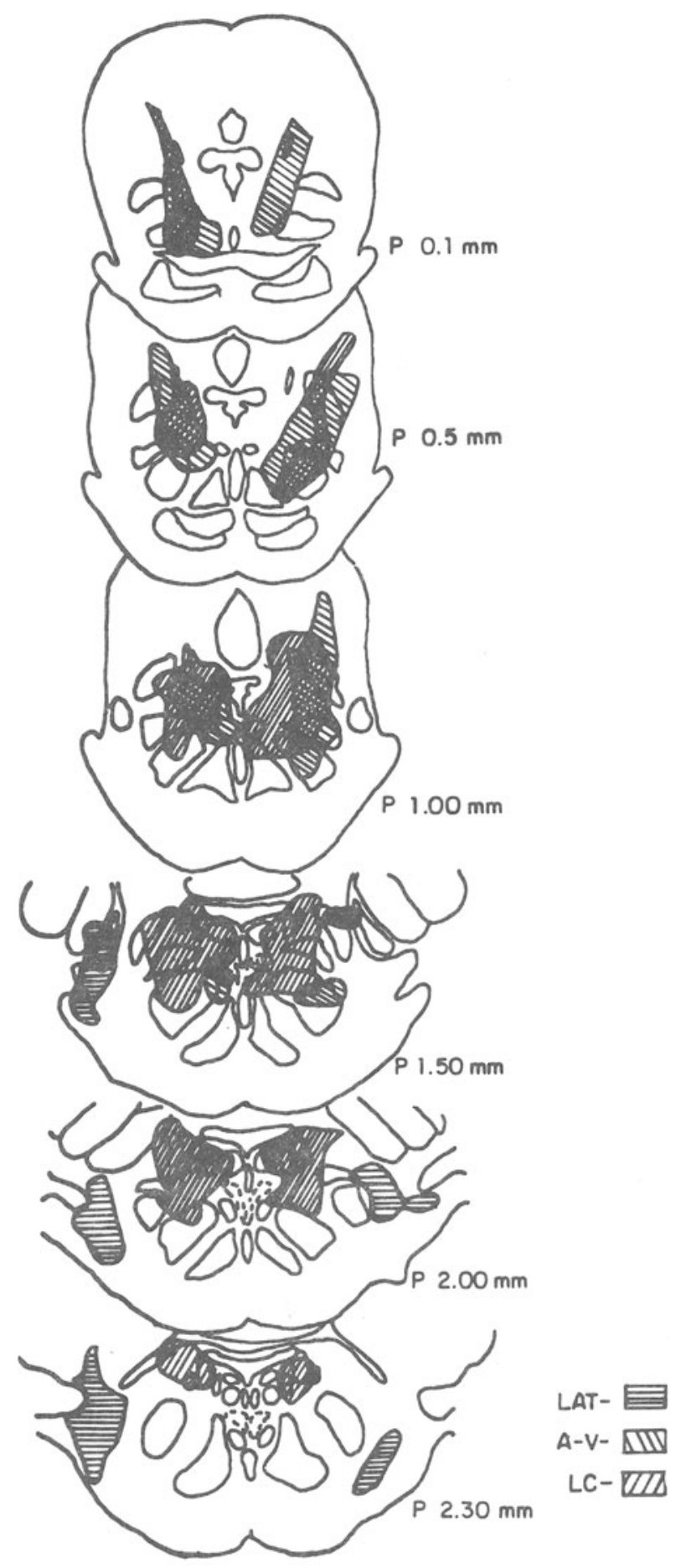

Figure 2. Schematic indicating the maximal extent of the lesions for all the rats in each group during Experiment 1. LC $=$ LClesioned group; $\mathbf{L A T}=$ group with lesions lateral to $\mathbf{L C} ; \mathbf{A}-\mathrm{V}=$ group lesioned anterior and ventral to $L C$. 
Table 1

Postlesion (PL) Changes From Baseline Conditions in Experiment 1

\begin{tabular}{|c|c|c|c|c|c|}
\hline Week & LC & Control & $\mathrm{F}$ & df & $\mathrm{p}<$ \\
\hline \multicolumn{6}{|c|}{ Intake (in Milliliters) } \\
\hline $\begin{array}{l}\text { PL1 } \\
\text { PL2 } \\
\text { PL3 }\end{array}$ & $\begin{array}{l}-3.41 \\
10.61 * \\
17.08 *\end{array}$ & $\begin{array}{r}-13.07 \\
-1.11 \\
-2.75\end{array}$ & $\begin{array}{r}1.95 \\
8.51 \\
36.06\end{array}$ & $\begin{array}{l}1,10 \\
1,10 \\
1,10\end{array}$ & $\begin{array}{l}\text { n.s. } \\
.05 \\
.0001\end{array}$ \\
\hline $\begin{array}{l}\text { PL1 } \\
\text { PL2 } \\
\text { PL3 }\end{array}$ & $\begin{array}{c}724.70 \\
4,562.00 \dagger \\
5,269.40\end{array}$ & $\begin{array}{r}\text { Licks } \\
-3,030.20 \\
-146.00 \\
-1,392.80\end{array}$ & $\begin{array}{r}8.92 \\
10.39 \\
16.71\end{array}$ & $\begin{array}{l}1,9 \\
1,9 \\
1,9\end{array}$ & $\begin{array}{l}.05 \\
.05 \\
.005\end{array}$ \\
\hline \multicolumn{6}{|c|}{ Meals per Day } \\
\hline $\begin{array}{l}\text { PL1 } \\
\text { PL2 } \\
\text { PL3 }\end{array}$ & $\begin{array}{l}6.85 \dagger \\
6.56 \\
6.53\end{array}$ & $\begin{array}{r}1.23 \\
-.46 \\
.48\end{array}$ & $\begin{array}{l}5.72 \\
6.61 \\
4.50\end{array}$ & $\begin{array}{l}1,9 \\
1,9 \\
1,9\end{array}$ & $\begin{array}{l}.05 \\
.05 \\
\text { n.s. }\end{array}$ \\
\hline \multicolumn{6}{|c|}{ Licks per Meal } \\
\hline $\begin{array}{l}\text { PL1 } \\
\text { PL2 } \\
\text { PL3 }\end{array}$ & $\begin{array}{l}-586.30 * * \\
-329.30 \\
-236.30\end{array}$ & $\begin{array}{r}317.00 \\
-\quad 113.00 \\
-\quad 137.70\end{array}$ & $\begin{array}{r}1.30 \\
2.14 \\
.18\end{array}$ & $\begin{array}{l}1,9 \\
1,9 \\
1,9\end{array}$ & $\begin{array}{l}\text { n.s. } \\
\text { n.s. } \\
\text { n.s. }\end{array}$ \\
\hline \multicolumn{6}{|c|}{ Weight (in Grams per Day) } \\
\hline $\begin{array}{l}\text { PL1 } \\
\text { PL2 } \\
\text { PL3 }\end{array}$ & $\begin{array}{r}-1.52 \\
.01 \\
2.79\end{array}$ & $\begin{array}{r}-.63 \\
.92 \\
-.96\end{array}$ & $\begin{array}{r}.57 \\
.28 \\
8.14\end{array}$ & $\begin{array}{l}1,10 \\
1,10 \\
1,10\end{array}$ & $\begin{array}{l}\text { n.s. } \\
\text { n.s. } \\
.05\end{array}$ \\
\hline
\end{tabular}

*Within-group difference from previous week $(p<.05)$. **Within-group difference from previous week $(p<.01)$. tWithin-group difference from previous week $(p<.005)$.

tween the 1st and 2nd weeks postlesion, and milk intake increased further during the 3rd week postlesion (see Table 1 and Figure 3).

Throughout the experiment more than $99 \%$ of all daily licks occurred during meals. During baseline conditions, the mean number of daily meals for all subjects was 9.2; the mean meal size was 1,422 licks.

The Hotelling-Lawley trace indicated no significant difference in the trends over time between the two groups for either meal frequency $[\mathrm{F}(3,7)=$ $2.53, \mathrm{p}>.10]$ or meal size $[\mathrm{F}(3,7)=1.54, \mathrm{p}>.10]$. As compared with controls, the LC-lesioned group had a significantly greater increase in meal frequency during the first 2 weeks postlesion, which fell just short of significance during the 3rd postlesion week. No differences occurred between the groups with respect to meal size. Within the LC-lesioned group, there was a significant increase in meal frequency and decrease in meal size from baseline to the 1st week postlesion. The decreased meal size gradually increased during subsequent weeks, while the number of meals remained elevated (see Table 1).

Increases in feeding were observed in the hyperphagic rats during both the light and dark parts of the cycle. There were no differences in the light/ dark distribution of feeding $[\mathrm{F}(1,10)=.06, \mathrm{p}>.10]$. Throughout the experiment, approximately $70 \%$ of all feeding occurred during the dark period.

The Hotelling-Lawley trace indicated significant $[F(3,8)=6.93, p<.05]$ differences between the groups in the rate (grams/day) of change of body weight over time. The LC-lesioned group significantly increased its rate of weight gain as compared with controls only during the 3rd week postlesion (see Table 1). At that time, however, they did not weigh significantly more than controls.

Changes in milk intake did not differ between the groups in response to quinine adulteration. All rats significantly reduced their intake during the first day of adulteration $[F(1,9)=26.45, p<.0001]$; this was followed by a gradual return to baseline feeding levels by Quinine Day 4.

Pearson product correlation coefficients were calculated to determine the relationships between changes in feeding behavior and the percent destruction to the LC, SC, DTN, and TMN. The changes in feeding behavior were measured by percent of baseline milk intake and number of licks during the 3rd week postlesion. As shown in Table 2, the magnitude of destruction to all structures except the DTN significantly correlated with changes in milk intake. The highest correlation was with SC destruction $(r=.79$, $p=.007)$. Interestingly, the change in number of licks correlated significantly with the magnitude of destruction to all the structures except the SC ( $r=$ $.49, \mathrm{p}>.10$ ). Destruction of the TMN correlated highest with changes in the number of licks $(r=.85$, $\mathrm{p}=.002)$.

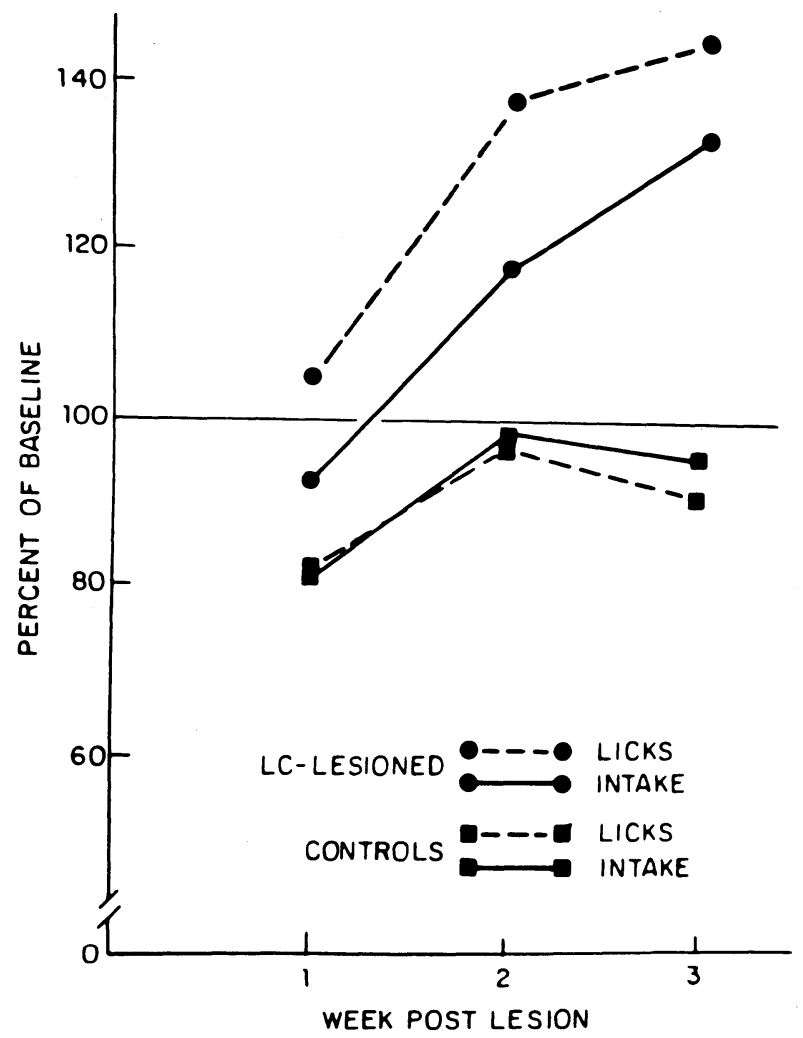

Figure 3. The mean percent of baseline milk intake and number of licks for LC-lesioned and control rats during Postlesion Weeks 1,2 , and 3. 
Table 2

Pearson Correlation Coefficients Between Feeding Changes and the Percentage of Destruction to Specific Nuclei During Experiment 1

\begin{tabular}{lccccc}
\hline & \multicolumn{2}{c}{ \% Baseline Intake } & & \multicolumn{2}{c}{ \% Baseline Licks } \\
\cline { 2 - 3 } & Pearson $\mathrm{r}$ & $\mathrm{p}$ & & Pearson $\mathrm{r}$ & $\mathrm{p}$ \\
\hline LC & .6709 & .034 & .8492 & .002 \\
DTN & .5028 & .139 & .7738 & .009 \\
TMN & .6896 & .027 & .8514 & .002 \\
SC & .7896 & .007 & .4938 & .147 \\
Baseline Intake & & & .8401 & .002 \\
\hline
\end{tabular}

\section{Discussion}

As predicted, the LC-lesioned group became hyperphagic. This increased feeding, however, was quite different from that found in HYP hyperphagia, in that no change in food intake occurred during the 1st week postlesion, the rats did not get obese, were not finicky, maintained their circadian feeding cycle, and increased their meal frequency rather than meal size.

Despite the fact that the rats were grouped based upon LC destruction, the magnitude of the lesions made it impossible to make a precise anatomical statement as to the area of destruction responsible for the feeding change. However, when all the lesions were superimposed upon one another (see Figure 2), there was no overlap between the LC-lesioned rats and the controls within the pontine dorsal tegmentum. Furthermore, correlational analyses indicate that destruction of the SC best predicted the magnitude of the change. TMN destruction best predicted enhanced licking, which, as suggested by Zeigler (1974), is probably due to a motor deficit.

Prior to Experiment 2, the histology was carefully analyzed and an area was selected that best represented the bilaterally destroyed loci in the hyperphagic rats that was intact, at least unilaterally, in all the controls. Figure 4A represents that area which coincides with much of the SC. The area actually includes the dorsal SC and extends medially, laterally, and ventrally to the LC, but does not include the dorsal portion of the nucleus. The histological data suggest that destruction in that area is responsible for the hyperphagia.

\section{EXPERIMENT 2}

Experiment 2 further explored the role of the dorsal hindbrain in feeding behavior by using: (1) a solidfood diet, to eliminate the possibility that the increased milk intake was due to hyperdipsia rather than hyperphagia; (2) smaller lesions, to make a more precise anatomical statement; and (3) cortical norepinephrine (NE) assays, to further examine the role of the LC in the hyperphagia. Furthermore, this experiment examined the possibility that the hyperphagic rats are hungrier than the controls, as sug- gested by the increased meal frequency and lack of finickiness, by utilizing meal pattern analyses, diet adulteration, and a task designed to assess motivation for food.

\section{Method}

Twenty-two rats, weighing approximately $330 \mathrm{~g}$, were food deprived for $24 \mathrm{~h}$ and then shaped to barpress for food pellets. They were housed individually in chambers with free access to water and a lever that delivered 45-mg Noyes pellets on a fixed-interval 5-sec schedule of reinforcement. This schedule was found to be effective in preventing the rats from hoarding pellets, thus allowing the measurement of meal patterns. A meal was defined as the acquisition of no less than 10 pellets separated by at least $10 \mathrm{~min}$ from the acquisition of any other pellets.

After 15 adaptation days, 20 rats were placed on a modified progressive ratio schedule of reinforcement for a 24 -h period. During this period, the rats were required to work progressively harder for pellets within a meal such that the first pellet was received after one barpress, the next pellet required two presses, and the third, three presses. This progression continued until the rat reached a fixed ratio-12 schedule of reinforcement which was maintained for the remainder of the meal. Upon termination of the meal $(10 \mathrm{~min}$ after the last pellet), the progression was reset back to the beginning.

Subsequent to 3 days of recovery, 7 days of baseline feeding behavior was recorded. Bilateral radio frequency lesions were then placed in the dorsal hindbrain of 18 rats; 4 received sham lesions. Twenty-one days of postlesion feeding behavior was recorded. The pellets were then adulterated with quinine (.12\% quinine) for 4 days in 16 rats. After 5 days of recovery, 20 rats were again placed on the progressive ratio schedule.

The rats were then sacrificed. The frontal cortices were removed and assayed for NE, using the spectrofluorometric assay technique described by Iwamoto, Loh, and Way (1976). Histology and localization of lesions were performed according to the same procedures used in Experiment 1. The rats were grouped for statistical analyses by the presence or absence of bilateral destruction within the area of the SC demarcated in Figure 4A. Rats with bilateral destruction within that area will be referred to as the SC group.

\section{Results}

Histology. Eleven of the 22 rats had bilateral destruction within the SC; the 11 controls consisted of 7 lesioned rats and 4 shams. Within the SC group, the subjects varied with respect to LC destruction (see Figures 4B and 4C). Four of the 7 lesioned controls had unilateral destruction within the SC; 1 of these had some bilateral LC destruction (see Figure 4D).

Behavioral analyses. No significant differences in feeding behavior occurred between the lesionedcontrols and shams; they were therefore combined to form the control group. Water intake was not recorded for two subjects because large amounts of spillage interfered with accurate measures during the prelesion condition. Table 3 summarizes the withinand between-group analyses for changes from baseline feeding during the 1st, 2nd, and 3rd weeks postlesion.

The Hotelling-Lawley trace indicated significant differences in the trends over time between the two groups for both food $[F(3,18)=10.63, p<.001]$ and water $[F(3,16)=6.21, p<.01]$ intake. As compared with controls, the SC group became significantly hy- 

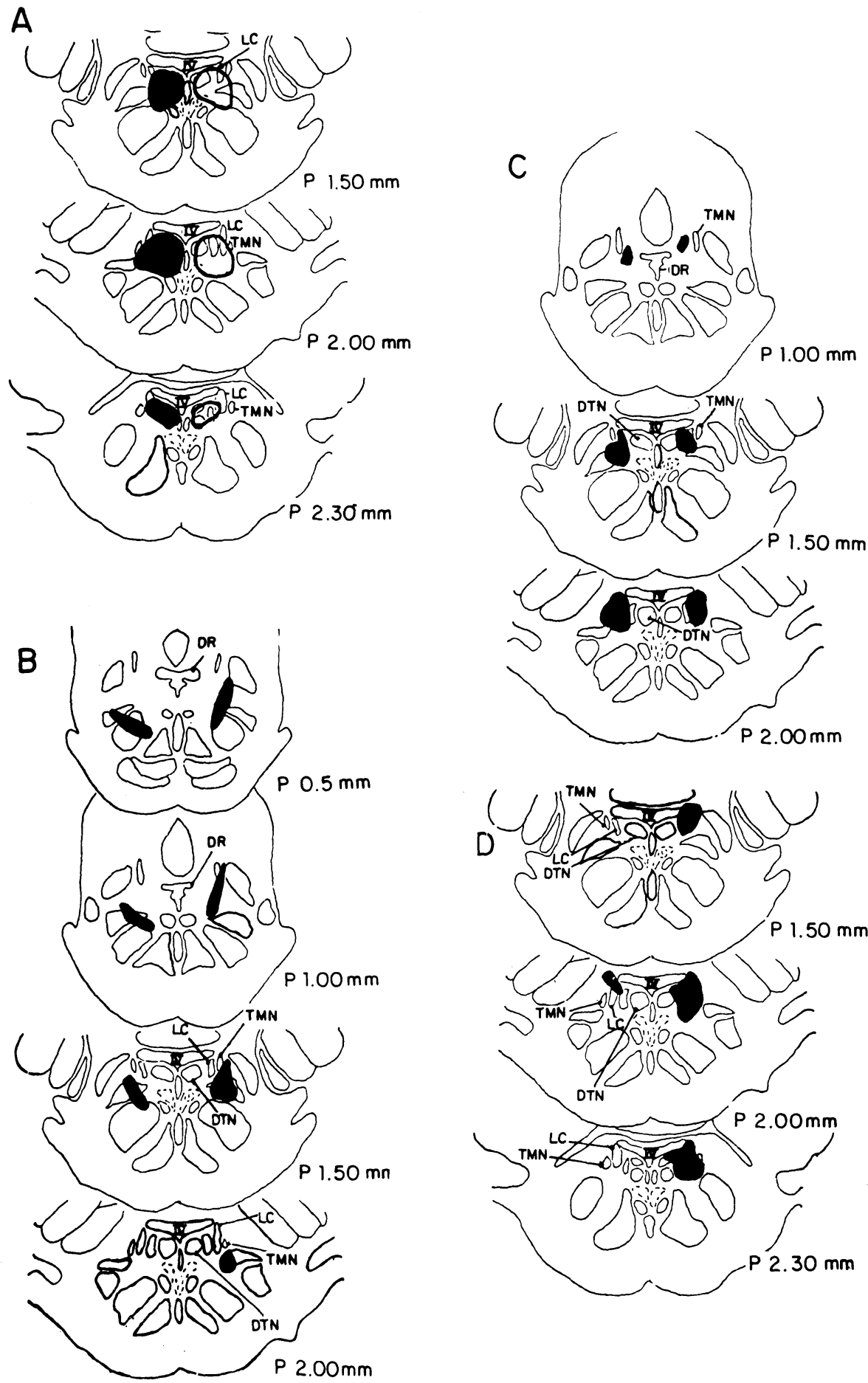

Figure 4. Schematic representation of (A) the area of destruction probably responsible for hyperphagia in Experiment 1, (B) bilateral SC lesions in rat 28L, (C) bilateral SC lesions in rat 30L, (D) unilateral SC lesion in rat $23 \mathrm{~L}$. LC = locus coeruleus; DTN = dorsal tegmental nucleus; TMN = trigeminal motor nucleus; DR = dorsal raphe; IV = fourth ventricle.

pophagic and hypodipsic during the 1st week postlesion; both of these measures returned to control levels during the 2 nd postlesion week. During the 3 rd week postlesion, the feeding of the SC rats increased significantly as compared with that of the controls, while their water intake remained at control levels. Within the SC group, both food and water intake decreased significantly between baseline and the 1st week postlesion, significantly increased between the 1 st and 2nd weeks postlesion, and did not alter sig- 
Table 3

Postlesion (PL) Changes From Baseline Conditions in Experiment 2

\begin{tabular}{|c|c|c|c|c|c|}
\hline Week & SC & Control & $\mathrm{F}$ & df & $\mathrm{p}<$ \\
\hline \multicolumn{6}{|c|}{ Intake (Number of Pellets) } \\
\hline $\begin{array}{l}\text { PL1 } \\
\text { PL2 } \\
\text { PL3 }\end{array}$ & $\begin{array}{c}-208.78 \dagger \\
47.40 \dagger \\
116.13\end{array}$ & $\begin{array}{l}-83.38 \\
-11.21 \\
-4.66\end{array}$ & $\begin{array}{r}11.16 \\
2.90 \\
10.77\end{array}$ & $\begin{array}{l}1,20 \\
1,20 \\
1,20\end{array}$ & $\begin{array}{l}.01 \\
\text { n.s. } \\
.01\end{array}$ \\
\hline \multicolumn{6}{|c|}{ Water (in Milliliters) } \\
\hline $\begin{array}{l}\text { PL1 } \\
\text { PL2 } \\
\text { PL3 }\end{array}$ & $\begin{array}{cc}-20.93 \dagger \\
-\quad 2.80 \dagger \\
2.95\end{array}$ & $\begin{array}{l}-9.04 \\
-2.77 \\
-1.17\end{array}$ & $\begin{array}{r}5.50 \\
.00 \\
.47\end{array}$ & $\begin{array}{l}1,18 \\
1,18 \\
1,18\end{array}$ & $\begin{array}{l}.05 \\
\text { n.s. } \\
\text { n.s. }\end{array}$ \\
\hline \multicolumn{6}{|c|}{ Meals per Day } \\
\hline $\begin{array}{l}\text { PL1 } \\
\text { PL2 } \\
\text { PL3 }\end{array}$ & $\begin{array}{ll}- & 7.20 \dagger \\
- & 3.60^{*} \\
- & 1.40 \dagger\end{array}$ & $\begin{array}{l}-3.70 \\
-1.90 \\
-1.10\end{array}$ & $\begin{array}{r}4.88 \\
1.31 \\
.04\end{array}$ & $\begin{array}{l}1,20 \\
1,20 \\
1,20\end{array}$ & $\begin{array}{l}.05 \\
\text { n.s. } \\
\text { n.s. }\end{array}$ \\
\hline \multicolumn{6}{|c|}{ Pellets per Meal } \\
\hline $\begin{array}{l}\text { PL1 } \\
\text { PL2 } \\
\text { PL3 }\end{array}$ & $\begin{array}{c}-\quad .50 \\
13.80^{*} \\
10.50\end{array}$ & $\begin{array}{l}1.00 \\
2.70 \\
1.00\end{array}$ & $\begin{array}{r}.14 \\
1.94 \\
2.10\end{array}$ & $\begin{array}{l}1,20 \\
1,20 \\
1,20\end{array}$ & $\begin{array}{l}\text { n.s. } \\
\text { n.s. } \\
\text { n.s. }\end{array}$ \\
\hline \multicolumn{6}{|c|}{ Nonmeal Pellets } \\
\hline $\begin{array}{l}\text { PL1 } \\
\text { PL2 } \\
\text { PL3 }\end{array}$ & $\begin{array}{l}19.50 \dagger \\
16.80 \\
14.70\end{array}$ & $\begin{array}{l}8.00 \\
2.80 \\
5.70\end{array}$ & $\begin{array}{l}1.29 \\
7.59 \\
6.26\end{array}$ & $\begin{array}{l}1,20 \\
1,20 \\
1,20\end{array}$ & $\begin{array}{l}\text { n.s. } \\
.05 \\
.05\end{array}$ \\
\hline \multicolumn{6}{|c|}{ Weight (in Grams per Day) } \\
\hline $\begin{array}{l}\text { PL1 } \\
\text { PL2 } \\
\text { PL3 }\end{array}$ & $\begin{array}{r}3.59 \\
3.26 \\
1.61\end{array}$ & $\begin{array}{r}1.53 \\
.84 \\
.32\end{array}$ & $\begin{array}{l}2.49 \\
8.41 \\
1.97\end{array}$ & $\begin{array}{l}1,20 \\
1,20 \\
1,20\end{array}$ & $\begin{array}{l}\text { n.s. } \\
.01 \\
\text { n.s. }\end{array}$ \\
\hline
\end{tabular}

*Within-group difference from previous week $(p<.05)$.

tWithin-group difference from previous week $(p<.005)$.

nificantly between the 2 nd and 3 rd weeks postlesion (see Table 3 and Figure 5).

Although the hyperphagic rats as a group did not become hyperdipsic as compared with controls, there was a significant correlation $(r=.72, p<.05)$ between their changes in food and water intake from baseline to the 3rd week postlesion. The change from baseline food/water ratio did not differ between the hyperphagic and control groups $[F(1,18)=1.64$, p > .10].

During baseline, $95.75 \%$ of all pellets were consumed during meals. The mean number of daily meals for all subjects was 16.1 ; the mean meal size was 34.25 pellets $(1.54 \mathrm{~g})$.

The Hotelling-Lawley trace indicated no significant difference in the trends over time between the two groups for meal frequency $[F(3,18)=2.12, p>$ $.10]$, meal size $[F(3,18)=2.08, p>.10]$, or the number of nonmeal pellets $[F(3,18)=2.61, p>.05]$. The hypophagia in the SC group during the 1st week postlesion was characterized by a significant decrease in meal frequency, as compared with controls which showed no changes in meal size or number of nonmeal pellets consumed. During the 2nd and 3rd weeks postlesion, the groups did not differ with respect to changes in meal frequency or meal size, but the SC group had a significantly greater increase in the number of nonmeal pellets.
Within the SC group, meal frequency significantly decreased from baseline to Postlesion Week 1 and then gradually returned to baseline levels. Meal size did not change between baseline and the 1st week postlesion, but increased significantly between the 1st and 2nd postlesion weeks; there were no further changes during the 3 rd postlesion week. The consumption of nonmeal pellets significantly increased from baseline to the 1st week postlesion, but did not vary during subsequent weeks (see Table 3 ).

Increases in feeding were observed in the hyperphagic rats during both the light and dark parts of the cycle, with no changes in the light/dark distribution of feeding $[\mathrm{F}(1,20)=.02, \mathrm{p}>.10]$. Approximately $70 \%$ of all feeding occurred during the dark period.

The Hotelling-Lawley trace indicated significant $[F(3,18)=5.72, p<.01]$ differences between the groups in the rate (grams/day) of change of body weight over time. While both groups lost weight during the 1st week postlesion, the rate of weight loss did not differ significantly between them. The SC group significantly increased its rate of weight gain as compared with controls during the 2 nd week postlesion. No differences occurred during the 3rd postlesion week (see Table 3 and Figure 5).

Changes in food intake did not differentially decrease between the groups in response to quinine adulteration. All rats significantly $[F(1,13)=89.76$, $\mathrm{p}<.0001]$ reduced their intake during the 1st day of adulteration and did not return to baseline levels by Quinine Day 4.

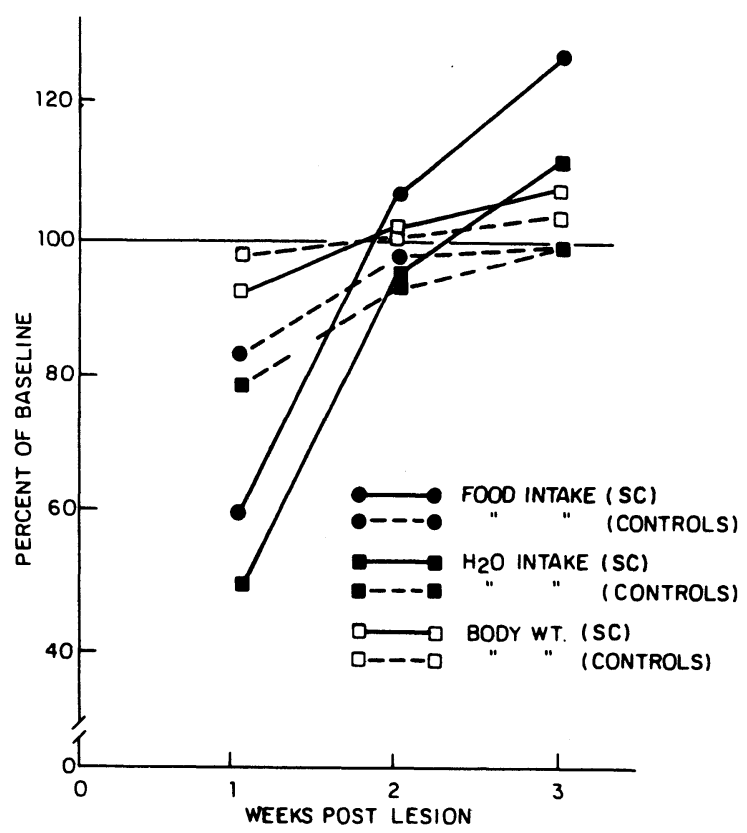

Figure 5. The mean percent of baseline food intake, water intake, and body weight for SC-lesioned and control rats during Postlesion Weeks 1, 2, and 3. 
Both groups decreased their pellet intake during the progressive ratio schedule in pre- and postlesion conditions. Prelesion, the decrease in intake from the previous day did not differ between groups $[t(18)=$ $.93, \mathrm{p}>.10]$. Postlesion, the SC-lesioned rats (which were now hyperphagic) had a significantly greater decrease in pellet intake $[t(18)=2.42, p<.05]$. The actual number of barpresses during the progressive ratio schedule did not differ between pre- and postlesion for either the SC group $[t(9)=.77, p>.10]$ or the controls $[\mathrm{t}(9)=1.47, \mathrm{p}>.10]$.

The SC rats had a $19 \%$ reduction in cortical $\mathrm{NE}$, while the lesioned controls had a $17 \%$ reduction as compared with that of the nonlesioned controls $[t(14)=$ $.04, \mathrm{p}>.10]$. Within the hyperphagic animals, there was no correlation between the magnitude of hyperphagia and the cortical NE depletion $(r=-.17$, $\mathrm{p}>.10)$.

Pearson product correlation coefficients between change in pellet intake and the magnitude of destruction to the LC, SC, TMN, and DTN indicated no significant correlations. As shown in Table 4, however, only the correlation between pellet intake and SC destruction approached significance $(p=.077)$.

\section{Discussion}

These data indicate that the increased milk intake in Experiment 1 was due to hyperphagia, rather than hyperdipsia. Although the food/water ratio was maintained in the SC-lesioned rats, they became significantly hyperphagic, but not hyperdipsic. The hyperphagia during the 3rd week postlesion was not a compensation for the decreased feeding during the 1 st week postlesion. This is indicated by the facts that (1) the SC group also ate more than did the controls during Postlesion Week 2, (2) the SC group weighed more than the controls at the end of the 2nd week postlesion, and (3) the SC group's increased food intake remained stable for about 9 days (see Figure 6). It is likely that the decreased eating during the 1st week postlesion was due to a nonspecific motor deficit, since (1) the rats appeared to have difficulty walking, (2) they did consume pellets when hand-fed, and (3) the rats in Experiment 1, in which feeding did not require a motor task, were not hypophagic.

Furthermore, the hyperphagia appears to be due to a satiety deficit, rather than to increased hunger, since the SC-lesioned rats did not work harder or

Table 4

Pearson Correlation Coefficients Between Food Intake Changes and the Percentage of Destruction to Nuclei in the Hindbrain

\begin{tabular}{lcc}
\hline & \multicolumn{2}{c}{ \% Baseline Intake } \\
\cline { 2 - 3 } & Pearson $\mathrm{r}$ & $\mathrm{p}$ \\
\hline LC & .1440 & .569 \\
DTN & .2272 & .365 \\
TMN & .1429 & .572 \\
SC & .4266 & .077 \\
\hline
\end{tabular}

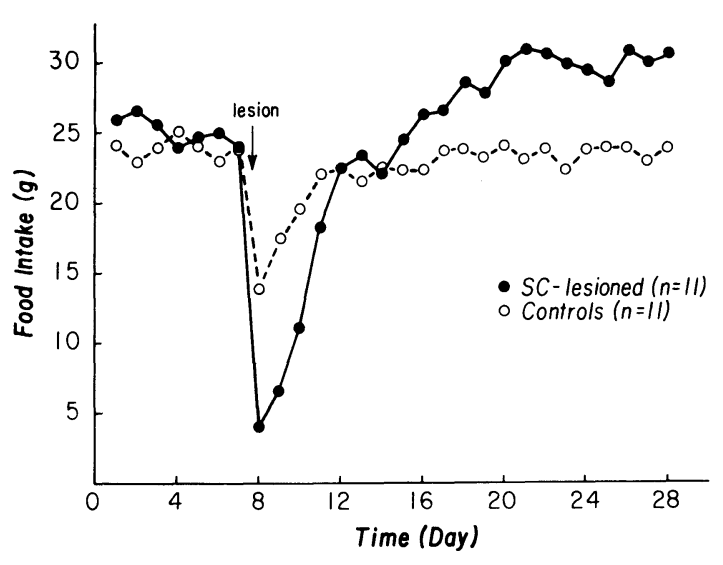

Figure 6. The mean daily food intake of SC-lesioned and control rats throughout Experiment 2.

tolerate quinine adulteration to maintain their increased food intake. The increased feeding was not reflected in changes of meal size or frequency as compared with controls, but rather increased "snacking"' (nonmeal pellets). Within the SC group, however, meal size increased between the 1st and 2nd weeks postlesion. Unlike the increased meal frequency observed in Experiment 1, this coincides with the onset of the hyperphagia and is also suggestive of a satiety deficit.

Histological analyses revealed that the area that best represented the bilaterally destroyed loci in the hyperphagic rats, which was intact at least unilaterally in the lesioned controls, was the dorsal part of the SC and the ventral tip of the LC. This coincides with data indicating that SC destruction best predicts the magnitude of the hyperphagia. The cortical NE assays further indicated that destruction of the principal LC is not responsible for the hyperphagia.

\section{GENERAL DISCUSSION}

The data indicate that lesions in the area of the SC result in a hyperphagia syndrome which is quite different from that following HYP lesions. SC-lesioned rats are less hyperphagic, are not obese, maintain their circadian feeding cycle, are not finicky, and do not overeat until about 1 week after the lesions.

The slow rise in body weight when compared with the magnitude of the hyperphagia is similar to that reported by Grossman and Grossman (1977) following knife cuts to the rostral brainstem. They suggest that it may be due to increased energy loss which is compensated for by the hyperphagia. In Experiment 1 , the number of licks increased faster than did milk intake, suggesting increased energy expenditure during feeding.

Unlike other hyperphagia syndromes which result from destruction of fibers entering the HYP, in which rats overeat immediately after the lesions, the rats in these studies did not become hyperphagic for at least 
1 week after the lesions. This time period coincides with that required for axonal degeneration, as indicated by the dopamine-beta hydroxylase reductions that occur in the HYP following hindbrain lesions (Ross \& Reis, 1974), suggesting that the effect may be due to alterations of HYP loci caused by the lesions rather than by a distinct hindbrain feeding system. Although the functional activity of central nervous system neurons during this time period is not known, in the peripheral nervous system it gradually decreases during axonal degeneration (Reis, Ross, \& Joh, 1974). An alternative explanation for the delayed onset of the hyperphagia is that it is due to denervation supersensitivity. We believe this to be less likely because it would imply that all effective lesions should initially result in decreased feeding. This did not occur in Experiment 1, during which no complex motor task was required for feeding. The initial hypophagia observed in Experiment 2 is similar to that found by others (Amaral \& Foss, 1975; Ross et al., 1973; Osumi et al., 1975) and is probably due to nonspecific behavioral suppression following the lesions for the reasons previously mentioned.

Although the hyperphagia syndrome resulting from SC lesions is quite different from that following HYP lesions, it has many similarities to the hyperphagia reported following lesions (Ahlskog, 1974; Ahlskog \& Hoebel, 1973) and knife cuts (Grossman \& Grossman, 1977) to the midbrain tegmentum. Rats with tegmentum destruction have significant HYP NE depletions, but the magnitude of the depletions is not related to the magnitude of the hyperphagia.

We hypothesize that the hyperphagia resulting from midbrain destruction, as well as that found in our studies, is due to denervation of some HYP nuclei via a pathway ascending from the SC. A NE pathway that connects these loci and ascends in close proximity to the ventral NE bundle (VNB) within the midbrain and posterior HYP has been previously described (Maeda \& Shimizu, 1972; Olson \& Fuxe, 1972). Because of the close proximity between this pathway and the VNB, lesions and knife cuts to the midbrain and posterior HYP are likely to disrupt both pathways. Destruction of this smaller pathway may account for much of the hyperphagia formerly attributed to VNB destruction.

This hypothesis, although tentative, pending more detailed neuroanatomical and neurochemical investigations, can explain why the magnitude of hyperphagia has not been found to correlate with HYP NE depletion, which is caused primarily by VNB destruction. Furthermore, it predicts that caudal VNB lesions (in the area of $A_{5}$ ), where this pathway is separated from the VNB, will result in significant HYP $\mathrm{NE}$ depletions, yet minimal alterations in feeding behavior.

\section{REFERENCES}

Aнцskog, E. Food intake and amphetamine anorexia after selective forebrain norepinephrine loss. Brain Research, 1974, 82, 211-240.

Ahlskog, J. E., \& Hoebel, B. G. Overeating and obesity from damage to a noradrenergic system in the brain. Science, 1973, 182, 166-169.

Amaral, D. G., \& Foss, J. A. Locus coeruleus lesions and learning. Science, 1975, 188, 377-378.

Amaral, D. G., \& Sinnamon, H. M. The locus coeruleus: Neurobiology of a central noradrenergic nucleus. Progress in Neurobiology, 1977, 9, 147-196.

Eison, M. S., Stark, A. D., \& Ellison, G. Opposed effects of locus coeruleus and substantia nigra lesions on social behavior in rat colonies. Pharmacology, Biochemistry and Behavior, 1977, 7, 87-90.

Goud, R. M. Hypothalamic obesity: The myth of the ventromedial nucleus. Science, 1973, 182, 488-490.

Graff, H., \& Stellar, E. Hyperphagia, obesity, and finickiness. Journal of Comparative and Physiological Psychology, 1962, $55,418-424$.

Grossman, S. P. Changes in food and water intake associated with an interruption of the anterior or posterior fiber connections of the hypothalamus. Journal of Comparative and Physiological Psychology, 1971, 75, 23-31.

Grossman, S. P., \& Grossman, L. Food and water intake in rats after transections of fibers en passage in the tegmentum. Physiology \& Behavior, 1977, 18, 647-658.

Iтамото, E. T., Loh, H. H., \& Way, E. L. Circling behavior after narcotic drugs and during naloxone precipitated abstinence in rats with unilateral nigral lesions. Journal of Pharmacology and Experimental Therapeutics, 1976, 197, 503-516.

Jones, B. E., \& Moore, R. Y. Ascending projections of the locus coeruleus in the rat. II. Autoradiographic study. Brain Research, 1977, 127, 23-53.

KIssileFF, H. R. Food-associated drinking in the rat. Journal of Comparative and Physiological Psychology, 1969, 67, 284-300.

Kluver, H., \& Barrera, E. A method for combined staining of cells and fibers in the nervous system. Journal of Neuropathology and Experimental Neurology, 1953, 12, 400-403.

Kobayashi, R. M., Palkovits, M., Jacobowitz, D. M., \& Kopin, I. Biochemical mapping of the noradrenergic projection from the locus coeruleus. Neurology, 1975, 25, 223-233.

Lindvall, O., \& BJorklund, A. The organization of the ascending catecholamine neuron systems in the rat brain as revealed by the glyoxylic acid fluorescence method. Acta Physiologica Scandinavica, 1974, Suppl. 412, 1-48.

Maeda, T., \& Shimizu, N. Projections ascendantes du locus coeruleus et d'autres neurones aminergiques pontiques au niveau du prosencephale du rat. Brain Research, 1972, 36, 19-35.

Morrison, D. F. Multivariate statistical methods (2nd ed.). New York: McGraw-Hill, 1976.

Olson, L., \& FuXE, K. Further mapping out of the central noradrenaline neuron systems: Projections of the "subcoeruleus" area. Brain Research, 1972, 43, 289-295.

Osumi, O., Oishi, R., Fujiwara, H., \& Takaori, S. Hyperdipsia induced by bilateral destruction of the locus coeruleus in rats. Brain Research, 1975, 86, 419-427.

Redmond, D. E., Huang, Y. H., Snyder, D. R., Maas, J. W., \& BAULU, J. Hyperphagia and hyperdipsia after locus coeruleus lesions in the stumptail monkey. Life Sciences, 1977, 20, 16191628.

REis, D. J., Ross, R. A., \& Joh, T. H. Some aspects of the reaction of central and peripheral noradrenergic neurons to injury. In K. Fuxe, L. Olson, \& Y. Zotterman (Eds.), Dynamics of degeneration and growth in neurons. Oxford: Pergamon Press, 1974. 
Ross, R. A., \& REIs, D. J. Effects of lesions of locus coeruleus on regional distribution of dopamine-beta-hydroxylase activity in the rat brain. Brain Research, 1974, 73, 161-166.

Ross, R. A., Smith, G. P., \& Reis, D. J. Effects of lesions of locus coeruleus on regional distribution of dopamine-betahydroxylase (DBH) in brain and feeding and drinking behaviors in rat. Federation Proceedings, 1973, 32, 708.

Sclafani, A. Neural pathways involved in the ventromedial hypothalamic lesion syndrome in the rat. Journal of Comparative and Physiological Psychology, 1971, 77, 70-96.
Teitelbaum, P. Disturbances in feeding and drinking behavior after hypothalamic lesions. In M. R. Jones (Ed.), Nebraska Symposium on Motivation. Lincoln: University of Nebraska Press, 1961.

Zeigler, H. P., \& Karten, H. J. Central trigeminal structures and the lateral hypothalamic syndrome in the rat. Science, 1974, 186, 636-638.

(Received for publication September 30, 1980; revision accepted April 17, 1981.) 